\title{
Association Between Microsatellite Instability and 18F-FDG PET/CT Data in Colorectal Cancer Patients
}

ZEKIYE HASBEK ( $\sim$ hasbekz@yahoo.com )

Cumhuriyet University

NECLA DEMIR

Acıbadem Kayseri Hospital,

MUKADDES YILMAZ

Cumhuriyet University

HATICE ÖZER

Cumhuriyet University

SEYIT AHMET ERTÜRK

Tokat Ministry of Health,

ÖZGE ULAŞ BABACAN

Cumhuriyet University

\section{Research Article}

Keywords: Microsatellite Instability, 18F-FDG PET/CT Data, Colorectal Cancer Patients

Posted Date: January 3rd, 2022

DOI: https://doi.org/10.21203/rs.3.rs-1213322/v1

License: (c) (i) This work is licensed under a Creative Commons Attribution 4.0 International License.

Read Full License 


\section{Abstract}

Aim: Our aim in this study was to evaluate the relationship between microsatellite instability (MSI) status and ${ }^{18} \mathrm{~F}-\mathrm{FDG}$ PET/CT data in patients with colorectal cancer.

Materials and Methods: Our study included 74 patients who underwent PET/CT in preoperative staging with the diagnosis of colorectal cancer and then underwent surgical resection. In the immunohistochemical examination, nuclear staining was considered positive for all antibodies. Normal colon mucosa and lymphocytes in tissue were used as an internal positive control group. The absence of nuclear staining in tumor cells was considered "loss" in "Mismatch Repair Gen" proteins. MSI status of patients was divided into 3 groups according to the occurrence of MLH1, PMS2, MSH2, MSH6 gene proteins, and also the number of MSI genes. It is defined as a high frequency of microsatellite instability (MSI-H) when two or more of the five markers in the tumor DNA were positive. If only one marker was positive, the tumor is termed as low frequency of MSI (MSI-L). And MSS is determined when all of the five markers were negative.

Results: While MSI was not detected in 56 of the patients (75.7\%), MSI was detected in 18 patients (24.3\%). 4 patients $(5.4 \%$ ) had MSH-L, while 14 patients $(18.9 \%)$ had MSH-H. In the analysis made considering all 3 groups, there was no statistically significant relationship between MSI status and SUVmax $(p=0.835)$. Liver metastases were present in $11(36.7 \%)$ of 30 patients who were metastatic at the time of diagnosis. Distant metastasis incidence was lower in ${ }^{18} \mathrm{~F}-\mathrm{FDG}$ PET/CT in patients with MSI-H $(p=0.010)$. In addition, a significant correlation was found between the presence of liver metastases and $\mathrm{MSI}$, and liver metastasis was not observed in any of the 14 patients with MSI-H $(p=0.041)$.

Conclusion: Although not statistically significant, SUVmax values were found to be higher in patients with $\mathrm{MSI}-\mathrm{H}$. In addition, metastasis rates were found to be lower in patients with microsatellite instability in accordance with the literature data.

\section{Aim}

Colorectal cancers are the third most common type of cancer worldwide (1). Colorectal cancer development is thought to occur through 2 different mutational pathways called chromosomal instability or microsatellite instability (2). Microsatellite instability (MSI) is caused by incompatibilities in the gene repair mechanism (Mismatch repair (MMR)) caused by DNA damage as a result of DNA replication errors, some of which lead to the accumulation of somatic changes in nucleotide repeat sequences called microsatellites, some of which occur in gene promoter regions (3). Patients with no unstable markers (MSS) are considered MSI stable. In the presence of microsatellite instability, MSI is grouped as low MSI (MSI-L) (1 marker) and high MSI (MSI-H) (2-5 markers) according to the instability levels of the markers examined (4). Microsatellite instability is genetically evaluated by comparing amplicons replicated by polymerized chain reaction (PCR) from DNA samples obtained from tumor cells with the MSI panel 
(primers targeting microsatellite sites). In addition to this test, DNA mismatch proteins can also be detected in cancerous tissue using an immunohistochemistry panel. Although PCR is the gold standard, DNA mismatch proteins can be detected in cancerous tissue using an immunohistochemistry panel other than PCR (5). The presence of microsatellite instability (MSI) is seen in $90 \%$ of hereditary Lynch syndrome colorectal cancer cases and $10-15 \%$ of sporadic colorectal cancers (6). The presence of MSI is observed in approximately $5 \%$ of patients with metastatic colorectal cancer (6). It is known that the efficacy of immunotherapy is lower in patients with microsatellite stable colorectal cancer (7). In addition, colorectal cancer patients with defective DNA mismatch repair mechanisms ("defective mismatch repair", dMMR) do not benefit from 5-FU adjuvant therapy, but on the contrary, they suffer from the side effects of the drug. For this reason, it is very important to determine the MSI or MMR status in terms of planning the treatment in patients who will be given adjuvant treatment (8).

Our aim in this study was to evaluate the relationship between MSI (microsatellite instability) status and ${ }^{18} \mathrm{~F}-\mathrm{FDG}$ PET/CT data in patients with colorectal cancer.

\section{Materials And Methods}

The study included 74 colorectal cancer patients who underwent PET/CT in preoperative staging and then underwent surgical resection in our hospital between 2018 and 2020. Patients who were operated on during this period but whose MSI status was not evaluated immunohistochemically were not included in the study. All of the patients provided written informed consent before undergoing the procedure, and this study (Number:2020-03/01) was approved by the local ethics committee, which confirmed that the study was in accordance with the ethical guidelines of the Helsinki Declaration.

\section{Immunohistochemical Analysis:}

Ready-to-use MSH2 (MutS protein homolog), MSH6 (forming MutSa), PMS2 (protein homolog), and MLH1 (MutL protein homologous) primary antibodies and DAB chromogen kit were used for immunohistochemical staining. In the immunohistochemical examination, nuclear staining was considered positive for all antibodies. Normal colon mucosa and lymphocytes in tissue were used as an internal positive control group. The absence of nuclear staining in tumor cells was considered "loss" in

"Mismatch Repair Gen" proteins. MSI status of patients was divided into 3 groups according to the occurrence of MLH1, PMS2, MSH2, MSH6 gene proteins, and also the number of MSI genes. It is defined as a high frequency of microsatellite instability (MSI-H) when two or more of the five markers in the tumor DNA were positive. If only one marker was positive, the tumor is termed as low frequency of MSI (MSI-L). And MSS is determined when all of the five markers were negative (9).

\section{${ }^{18}$ F-FDG PET/CT Imaging Procedure, Acquisitions, and Analysis:}


${ }^{18} \mathrm{~F}-\mathrm{FDG}$ PET/CT imaging was performed in the Department of Nuclear Medicine with a combined PET/CT scanner (Discovery 600 PET/CT GE Medical Systems, USA). According to our routine imaging protocol each patient fasted for at least $6 \mathrm{~h}$ before imaging and after ensuring that blood glucose was $<200 \mathrm{mg} / \mathrm{dL}$, approximately $10 \mathrm{mCi}{ }^{18} \mathrm{~F}-\mathrm{FDG}$ was administered intravenously 1 hour before image acquisition. Patients with fasting blood glucose above $200 \mathrm{mg} / \mathrm{dl}$ were canceled with the recommendation of blood glucose regulation to be taken at another date. After the injection, all patients were kept in the restroom for approximately 45-60 minutes. CT imaging was performed with a spiral 16slice scanner at $120 \mathrm{kV}, 172 \mathrm{mAs}$ for attenuation correction and anatomical correlation. A standard PET imaging protocol was taken from the cranium to the mid-thigh with an acquisition time of $2 \mathrm{~min} / \mathrm{bed}$ in 3dimensional mode. All PET studies were acquired in 3-D mode. CT images were acquired with $70 \mathrm{~mA}$, $120 \mathrm{kV}$, axial slice thickness of $2.5 \mathrm{~mm}$. Axial, coronal, and sagittal fusion images were created using the iterative reconstruction method. And then the visual and semi-quantitative analyses were performed respectively. The Maximum Standardized Uptake Value (SUVmax) was calculated from the obtained PET images. The standardized uptake value is a measurement normalized to body weight/surface area and injected activity. The standardized uptake value (SUV) was calculated by the following formula:

[Activity of ROI (mCi / ml) $\times$ Bodyweight (gram) $] \div$ Injected dose $(\mathrm{mCi})$

\section{Statistical analysis:}

The obtained data were evaluated with the SPSS 23.0 program (Statistical Package for the Social Sciences, SPSS Inc., Chicago). The normality of the data was examined by the Kolmogorov-Smirnov test. If the data provided the parametric conditions, they were analyzed with the independent sample t-test for two independent groups and the $F$ test (ANOVA) for more than two groups. While using ANOVA for comparisons with more than two groups, Tukey tests were used for those who provided the homogeneity assumption and Tamhane's T2 tests were used for those who did not provide the assumption of homogeneity to determine which group was different from the others. If any or all of the assumptions were not met, the Mann Whitney $U$ test was used for two independent groups and the Kruskal Wallis test was used for more than two independent groups. A Chi-square test was used to evaluate the data obtained by counting. The level of error was taken as 0.05 .

\section{Results}

All of the 74 patients (median age: 63; range: $32-89$ years) included in the study, including 23 women $(31.1 \%)$ and 51 men (68.9\%), had adenocarcinoma. The tumor was located in the rectum in 23 patients $(31.1 \%)$, rectosigmoid in 6 patients $(8.1 \%)$, and colon in 45 patients $(60.8 \%)$. Seven patients $(9.5 \%)$ were Stage I, 16 (21.6\%) Stage IIA, 4 (5.4\%) Stage IIB, 1 (1.4\%) Stage IIIA, 10 (13.5\%) Stage IIIB , 6 (8.1\%) were Stage IIIC and 30 (40.5\%) were Stage IV. The median value of the largest tumor size was $4.25 \mathrm{~cm}$ (range: 1-12 cm). The median SUVmax value measured from the primary tumor localization was 14 (range: 646). 
While there was metastasis in 30 patients $(40.5 \%)$ at the time of diagnosis, there was no metastasis in 44 patients (59.5\%). The tumor was located in the colon in 17 (56.7\%) and in the rectosigmoid in 13 (43.3\%) of the metastatic patients. Eleven (36.7\%) of 30 metastatic patients had liver metastases. Patients with $\mathrm{MSI}-\mathrm{H}$ had a lower incidence of distant metastases in ${ }^{18} \mathrm{~F}-\mathrm{FDG}$ PET/CT $(p=0.010)$. There was also a significant relationship between the presence of liver metastases and MSI, and none of the 14 patients with MSI-H had liver metastasis $(p=0.041)$ (Table 1).

\section{Table 1}

The relationship between microsatellite instability (MSI) status and distant metastases and liver metastases. MSS (without MSI), MSI-L (with MSI in 1 gene), MSI-H (with MSI in 2-5 genes).

\begin{tabular}{|c|c|c|c|c|c|}
\hline & & \multicolumn{4}{|c|}{ MSI Status } \\
\hline & & MSS & MSI-L & MSI-H & $p$ value \\
\hline & & $\mathrm{n}(\%)$ & n (\%) & $\mathrm{n}(\%)$ & \\
\hline \multirow[t]{2}{*}{ Distant metastasis } & Present & $26(86.7)$ & $3(10)$ & $1(3.3)$ & \\
\hline & Absent & $30(68.2)$ & $1(2.3)$ & $13(29.5)$ & $0.010 *$ \\
\hline \multirow[t]{2}{*}{ Liver metastasis } & Present & $9(81.8)$ & $2(18.2)$ & $0(0.0)$ & $0.041^{*}$ \\
\hline & Absent & $47(74.6)$ & $2(3.2)$ & $14(22.2)$ & \\
\hline
\end{tabular}

MSI was not seen in 56 of the patients (75.7\%). MSI was present in 18 patients (24.3\%). MSI was low (MSH-L) in 4 patients (5.4\%), and high (MSH-H) in 14 patients (18.9\%).In the analysis made considering all 3 groups, there was no statistically significant relationship between MSI status and SUVmax $(p=0.835)$. The median SUVmax value was found to be 12 (range: 8-28) in patients with MSI-H, 14 (range: 10-29) in patients with MSI-L, and 14.75 (range: 6-46) in patients with MSS.

$13.5 \%$ of our patients had a family history of colorectal cancer. However, no statistically significant correlation was found between family history of colorectal cancer and MSI $(p=0.085)$. In addition, there was no significant correlation between MSI status and histopathological findings of the tumor such as vascular invasion, venous invasion, perineural invasion, tumor budding, lymphatic invasion $(p=0.266 ; 0.196 ; 0.063 ; 0.811 ; 0.16$, respectively). No significant relationship was found between MSI and age, gender, and stage ( $p=0.065,0.415$, and 0.485 , respectively) (TableXX). However, MSI-H was most commonly present in stage II disease. Of the patients with MSI-H, 2 (14.3\%) were Stage I, 6 (42.9\%) Stage II, 5 (35.7\%) Stage III, and 1 (7.1\%) Stage IV.

A significant correlation was found between the largest tumor size and MSI status ( $p=0.017)$. While the median tumor size was $4 \mathrm{~cm}$ in patients with MSS and MSI-L, the median tumor size was $7 \mathrm{~cm}$ in patients with MSI-H.

\section{Discussion}


Colorectal cancers are the 3rd most common cancer type after lung and prostate cancer in men and the second most common cancer type after breast cancer in women (10). Genetic predispositions such as familial adenomatous polyposis syndrome, hereditary non-polyposis colorectal cancer, family history (especially cases younger than 60 years old) are important for the development of colorectal cancer. The prognosis and choice of treatment are determined by the tumor stage at the time of diagnosis. Therefore, imaging plays an important role in staging. CT and MRI are important in preoperative staging (especially T staging). In clinical practice, ${ }^{18} \mathrm{~F}-\mathrm{FDG} \mathrm{PET} / \mathrm{CT}$ is rarely used in the primary diagnosis of colorectal cancers. However, it has high sensitivity and specificity in the evaluation of distant metastases and also for postoperative surveillance $(11,12) .{ }^{18} \mathrm{~F}-\mathrm{FDG}$ PET/CT provides mainly metabolic information apart from tumor anatomy. Increased FDG uptake is associated with an aggressive tumor pattern and poor prognosis (13). According to the study of Tessem et al. (14) in colorectal cancer tissues, lactate, glycine, taurine, creatine, PC, and most of the metabolites of free choline, GPC, Myo-inositol, and glucose were found to be lower in MSI-H tissue samples compared to MSS. Perhaps it is based on the fact that altered metabolites in cancer tissues are associated with expected metabolic disturbances such as glycolysis, hypoxia, nucleotide biosynthesis, lipid metabolism, inflammation, and steroid metabolism, and perhaps this may explain the different FDG uptake levels in MSS and MSI tissues. However, there is a need for further studies investigating the changes in signal transduction pathways that lead to increased glycolytic activity in MSI tumors.

The relationship with family history in colorectal cancers was noticed many years ago (Lynch syndrome), and then differences in DNA repair genes were detected in these tumors. However, it is known that MSI is associated with sporadic cases as well as familial syndromes in colorectal cancers (15). The mechanism that causes MSI is the loss of proteins responsible for the DNA mismatch repair mechanism.

Microsatellite instability is genetically evaluated by comparing amplicons replicated by polymerized chain reaction (PCR) from DNA samples obtained from tumor cells with the MSI panel (primers targeting microsatellite sites). In addition, DNA mismatch repair proteins (MLH1, PMS2, MSH2, and MSH6; IHC panel) can be studied immunohistochemically in the cancerous tissue itself, and positive nuclear staining or loss can be observed in cancer cells (16) (Figure 1,2). The majority of hereditary nonpolyposis colorectal cancer occurs due to MLH1 or MSH2 mutations (2). The NCCN guideline recommends examining the microsatellite instability in CRC patients (17). Detecting MMR deficiency in colorectal cancers is important for prognosis. MSI-H CRC patients have a better prognosis and antitumor immune response than MSS patients $(18,19)$. Although there are controversial data in the literature, patients with high MSI in non-metastatic colorectal cancers are associated with a lower risk of recurrence and higher survival than those without MSI. According to studies, the prognosis of MSI-H tumors is worse in metastatic patients (20).

In our study, MSI-H was most frequently present in stage II disease. Of the patients with MSI-H, 2 (14.3\%) were Stage I, 6 (42.9\%) Stage II, 5 (35.7\%) Stage III, and 1 (7.1\%) Stage IV. According to the study of Mohan et al. (21), the rate of metastasis was found to be lower in Stage I / II CRC patients. In our study, 
none of the 6 patients with stage I and 21 patients with stage II had distant metastases. Among patients with MSI-H, only 1 patient had distant metastases at the time of diagnosis (Table 1).

It is known that MSI-H colorectal cancers are mostly located in the proximal colon. According to a study, in $2 / 3$ of HNPCC patients and more than $90 \%$ of patients with MSI-H sporadic colorectal cancer, the lesion is detected proximal to the splenic flexure (22). In our study, our patients with MSI-H were located in the proximal colon (Table 2), consistent with these literature data.

\section{Table 2}

Relationship between MSI (microsatellite instability) status and stage, gender and age. MSS (without MSI), MSI-L (with MSI in 1 gene), MSI-H (with MSI in 2-5 genes).

\begin{tabular}{|c|c|c|c|c|c|}
\hline & \multicolumn{4}{|c|}{ MSI Status } \\
\hline & & MSS & MSI-L & MSI-H & $p$ value \\
\hline & & $\mathrm{n}(\%)$ & n (\%) & n (\%) & \\
\hline \multirow{4}{*}{ Stage } & $I(n=6)$ & $4(66.7)$ & - & $2(33.3)$ & \multirow{4}{*}{0.485} \\
\hline & II $(n=21)$ & $15(71.4)$ & - & $6(28.6)$ & \\
\hline & III $(n=17)$ & $11(64.7)$ & $1(5.9)$ & $5(29.4)$ & \\
\hline & IV $(n=30)$ & $26(86.7)$ & $3(10)$ & $1(3.3)$ & \\
\hline \multirow[b]{2}{*}{ Localisation } & Colon & $30(66.7)$ & $1(2.2)$ & $14(31.1)$ & \multirow[b]{2}{*}{$0.02^{\star}$} \\
\hline & Rectosigmoid colon & $26(89.7)$ & $3(10.3)$ & $0(0)$ & \\
\hline \multirow[t]{2}{*}{ Gender } & Female & $20(87)$ & - & $3(13)$ & \multirow[t]{2}{*}{0.226} \\
\hline & Male & $36(70.6)$ & 4 /7.8) & $11(21.6)$ & \\
\hline \multirow[t]{2}{*}{ Age } & $<70$ & $38(79.2)$ & $386.3)$ & $7(14.6)$ & \multirow[t]{2}{*}{0.415} \\
\hline & $\geq 70$ & $18(69.2)$ & $1(3.8)$ & $7(26.9)$ & \\
\hline \multicolumn{2}{|c|}{ Median Tumor size } & $4 \mathrm{~cm}$ & $4 \mathrm{~cm}$ & $7 \mathrm{~cm}$ & $0.010 *$ \\
\hline
\end{tabular}

According to our study, the tumor size was found to be larger in patients with MSI-H.

In a meta-analysis by Wang et al. (23) in which they estimated the cumulative risk of colorectal cancer in heterozygous mutation carriers by age and sex, they estimated the cumulative risk of colorectal cancer by age and sex in heterozygous mutation carriers. According to this study, At age 70 years, for male and female carriers, respectively, risks for $M L H 1$ were $43.9 \%$ and $37.3 \%$, for $M S H 2$ were $53.9 \%$ and $38.6 \%$, and for $\mathrm{MSH} 6$ were $12.0 \%$ and $12.3 \%$. In the study of Rigau et al. (24), although it was reported that patients 
with MLH1 gene loss were older and patients with MSH2 gene loss were younger, no significant relationship was found between $\mathrm{MSI}$ and age in our study.

The biggest limitation of our study was that it was retrospective, with heterogeneous patient groups and a limited number of patients. In the future, there is a need for studies involving more patients and to be conducted with different institutions.

As a result, in our study, SUVmax values were found to be higher in patients with MSI-H, although it was not statistically significant. In addition, in accordance with the literature data, the rate of metastasis was found to be lower in patients with MSI. Although a positive and significant relationship was reported between MSI and SUVmax values in gastric cancers in our literature review (25), we think that the results of our study are valuable since no study was found that evaluated MSI and PET/CT data in colorectal cancers.

\section{Conclusion}

Metastasis rates were found to be lower in patients with MSI. Although SUVmax values were high in patients with MSI-H, there was no statistical significance between these two. However, new studies with a larger number of patients are needed.

\section{Declarations}

\section{Ethics}

All of the patients provided written informed consent before undergoing the procedure, and this study (Number:2020-03/01) was approved by the local ethics committee, which confirmed that the study was in accordance with the ethical guidelines of the Helsinki Declaration.

\section{Conflict of Interest}

The authors have no conflicts of interest to declare regarding this study.

\section{Authors' contributions}

Z.H. and N.D. conceived and designed the experiments. H.Ö. performed the experiments. Z.H., M.Y., H.Ö. and Ö.U:B. Provided patient selection and access to patient information. Z.H analyzed the data and wrote the paper. S.A.E. translated the article into English. All authors read and approved the fnal manuscript.

\section{Consent for publication}




\section{Competing interests}

The authors declare that there is no confict of interest.

\section{Funding}

Not applicable

\section{Acknowledgements}

Not applicable.

\section{References}

1. Kolligs FT. Diagnostics and epidemiology of colorectal cancer. Visc Med 2016; 32:158-64.

2. Gryfe R. Overview of colorectal cancer genetics. Surg Oncol Clin N Am . 2009;18(4):573-83.

3. Brammer DW, Gillespie PJ, Tian M, Young D, Raveendran M, Williams LE, Gagea M, et al. MLH1rheMac hereditary nonpolyposis colorectal cancer syndrome in rhesus macaques. Proc Natl Acad Sci U S A. 2018.13;115(11):2806-2811. doi: 10.1073/pnas.1722106115.

4. Cheah, P. L., Jing, L. I., Lai-Meng, L. O. O. I., Cing-Chai, K. O. H., Tze-Pheng, L. A. U., Chang, S. W. Screening for microsatellite instability in colorectal carcinoma: Practical utility of immunohistochemistry and PCR with fragment analysis in a diagnostic histopathology setting. Malaysian Journal of Pathology. 2019,41(2).

5. Bacher, JW., Flanagan, LA., Smalley, RL., Nassif, NA., Burgart, LJ., Halberg, R. B.,et al. Development of a fluorescent multiplex assay for detection of MSI-High tumors. Disease markers, 2004, 20.4, 5: $237-$ 250.

6. Tougeron D, Sueur B1, Zaanan A, De La Fouchardiere C, Sefrioui D, et al. Prognosis and Chemosensitivity of Deficient MMR Phenotype in Patients with Metastatic Colorectal Cancer: an AGEO Retrospective Multicenter Study. Int J Cancer. 2020 Jan 22. doi: 10.1002/ijc.32879.

7. Lee MKC, Loree JM. Current and emerging biomarkers in metastatic colorectal cancer. Curr Oncol. 2019;26(Suppl 1):S7-S15.

8. Sargent DJ, Marsoni S, Monges G, Thibodeau SN, Labianca R, Hamilton SR, et al. Defective mismatch repair as a predictive marker for lack of efficacy of fluorouracil-based adjuvant therapy in colon cancer. J Clin Oncol. 2010,10;28(20):3219-26.

9. Yuan L, Chi Y, Chen W, Chen X, Wei P, Sheng W., et al. Immunohistochemistry and microsatellite instability analysis in molecular subtyping of colorectal carcinoma based on mismatch repair 
competency. Int J Clin Exp Med 2015;8(11):20988-21000.

10. Bray F, Ferlay J, Soerjomataram I, et al. Global cancer statistics 2018: GLOBOCAN estimates of incidence and mortality worldwide for 36 cancers in 185 countries. CA Cancer J Clin, 2018; 68: 394424.

11. Monteil J, Le Brun-LyV, Cachin F, Zasadny X, Seitz JF, Mundler O, et al. Comparison of 18FDG$\mathrm{PET} / \mathrm{CT}$ and conventional follow-up methods in colorectal cancer: A randomised prospective study. Digestive and Liver Disease 53 (2021) 231-237.

12. Shin SS, Jeong YY, Min JJ, Kim HR, Chung TW, Kang HK. Preoperative staging of colorectal cancer: CT vs. integrated FDG PET/CT. Abdom Imaging. 2008;33(3):270-7.

13. Chen R, Wang Y, Zhou X, Huang G, Liu J. Preoperative PET/CT 18 F-FDG Standardized Uptake by Lymph Nodes as a Significant Prognostic Factor in Patients with Colorectal Cancer. Contrast Media Mol Imaging. 2018.1;2018:5802109.

14. Tessem MB, Selnaes KM, Sjursen W, Tranø G, Giskeødegård GF, Bathen TF, Gribbestad IS, Hofsli E. Discrimination of patients with microsatellite instability colon cancer using 1H HR MAS MR spectroscopy and chemometric analysis. J Proteome Res . 2010.2;9(7):3664-70.

15. Boland CR, Goel A. Microsatellite instability in colorectal cancer. Gastroenterology. Haziran 2010;138(6):2073-87.e3.

16. Luchini C, Bibeau F, Ligtenberg MJL, Singh N, Nottegar A, Bosse T, et al. ESMO recommendations on microsatellite instability testing for immunotherapy in cancer, and its relationship with PD-1/PD-L1 expression and tumour mutational burden: a systematic review-based approach. Annals of Oncology 2019, 30: 1232-1243.

17. Provenzale D, Ness RM, Llor X, Weiss JM, Abbadessa B, Cooper G, Early DS, Friedman M, Giardiello FM, et al. NCCN Guidelines: Colorectal Cancer Screening, Version 2.2021.

18. Marginean EC, Melosky B. Is There a Role for Programmed Death Ligand-1 Testing and Immunotherapy in Colorectal Cancer With Microsatellite Instability? Part I-Colorectal Cancer: Microsatellite Instability, Testing, and Clinical Implications. Arch Pathol Lab Med. 2018 Jan;142(1):17-25.

19. Yang G, Zheng RY, Jin ZS. Correlations between microsatellite instability and the biological behaviour of tumours. J Cancer Res Clin Oncol. 2019;145(12):2891-2899.

20. Aasebø Kø, Dragomir A, Sundström M, Mezheyeuski A, Edqvist PH, et al. Consequences of a high incidence of microsatellite instability and BRAF-mutated tumors: A population-based cohort of metastatic colorectal cancer patients. Cancer Med. 2019 ;8(7):3623-3635.

21. Mohan HM, Ryan E, Balasubramanian I, Kennelly R, Geraghty R, Sclafani F, et al. Microsatellite instability is associated with reduced disease specific survival in stage III colon cancer. Eur J Surg Oncol. 201;42(11):1680-1686.

22. Smedt LD, Lemahieu J, Palmans S, Govaere O, Tousseyn T, Cutsem EV, et al. Microsatellite instable vs stable colon carcinomas: analysis of tumour heterogeneity, inflammation and angiogenesisBritish Journal of Cancer (2015) 113, 500-509. 
23. Wang C, Wang Y, Hughes KS, Parmigiani G, Braun D. Penetrance of Colorectal Cancer Among Mismatch Repair Gene Mutation Carriers: A Meta-Analysis. JNCI Cancer Spectr. 2020.23;4(5):pkaa027.

24. Rigau V, Sebbagh N, Olschwang S, Paraf F, Mourra N, Parc Y, Flejou JF. Microsatellite instability in colorectal carcinoma. The comparison of immunohistochemistry and molecular biology suggests a role for hMSH6 [correction of hMLH6] immunostaining. Arch Pathol Lab Med. 2003;127(6):694-700.

25. Chung HW, Lee SY, Han HS, Park HS, Yang JH, Lee HH, So Y. Gastric cancers with microsatellite instability exhibit high fluorodeoxyglucose uptake on positron emission tomography. Gastric Cancer. 2013;16(2):185-92.

\section{Figures}

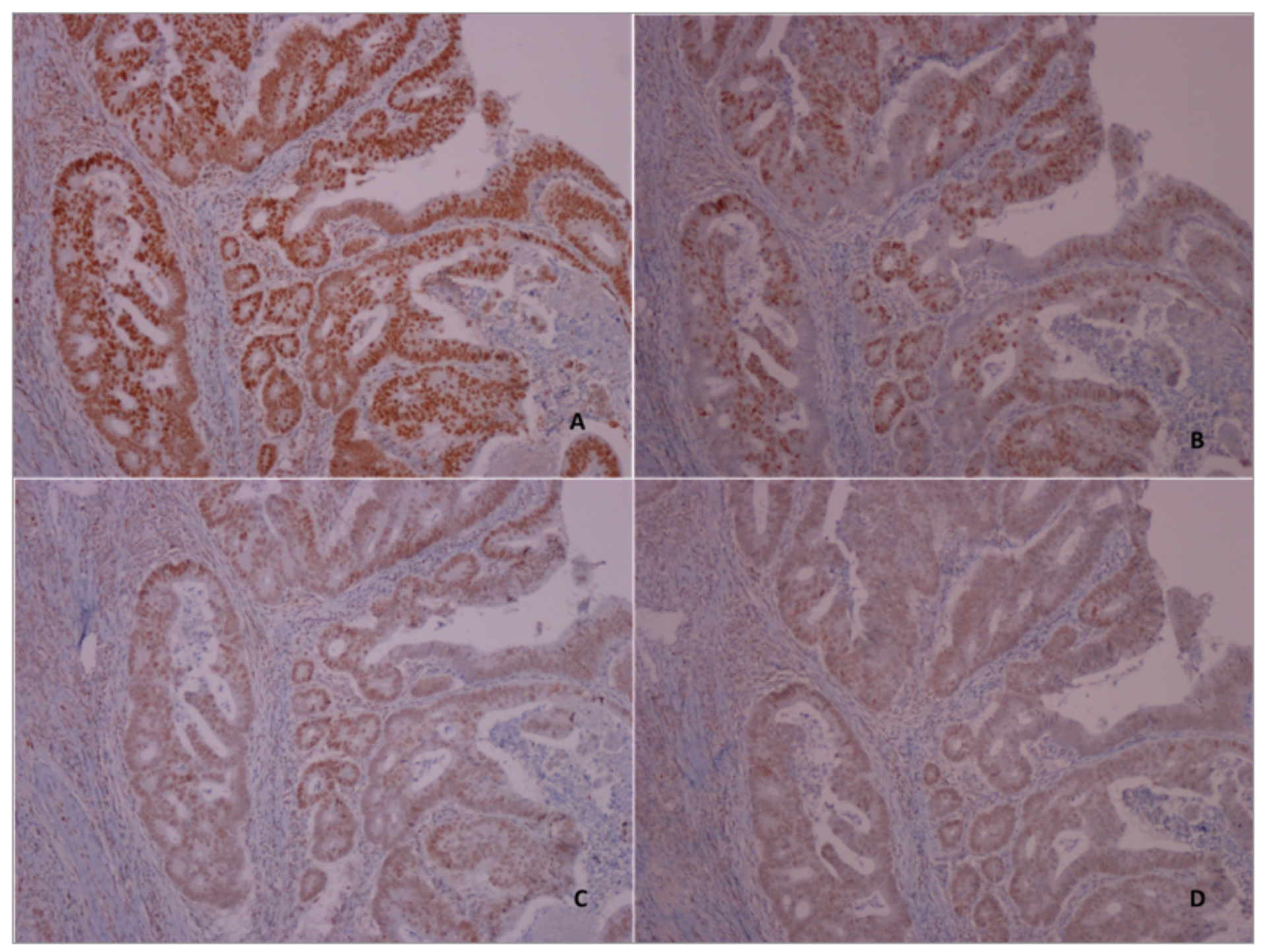

\section{Figure 1}


No loss of nuclear expression was observed in any of the MMR proteins in the immunohistochemical analysis. (A: MSH2;X100) (B: MSH6;X100) (C: MLH1;X100) (D:PMS2;X100)

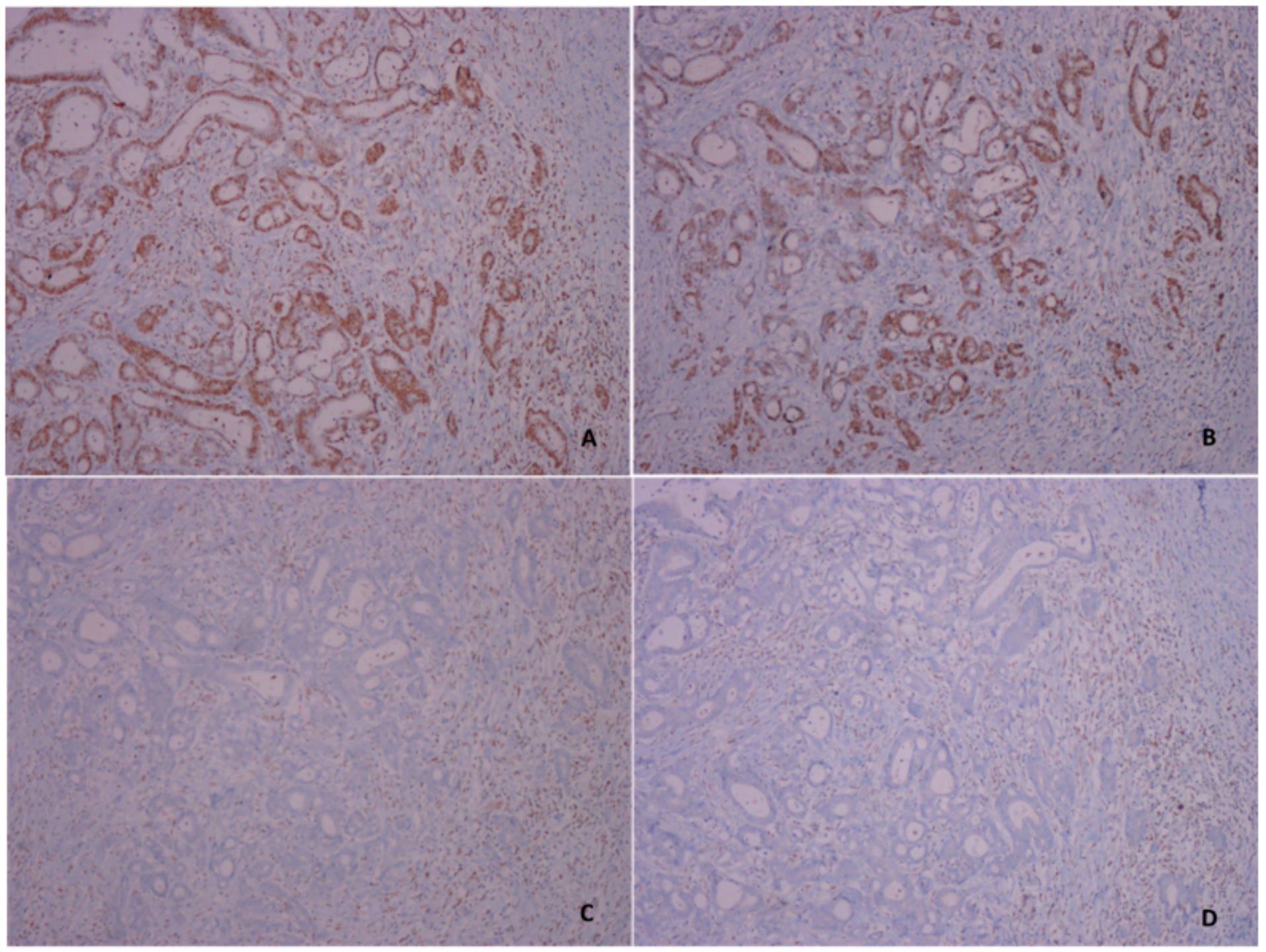

\section{Figure 2}

Immunohistochemical analysis showed no loss of nuclear expression with $\mathrm{MSH} 2$ and $\mathrm{MSH} 6$ in tumor cells, whereas nuclear expression loss was present with MLH1 and PMS2. (A: MSH2;X100) (B: MSH62;X100) (C: MLH1;X100) (D:PMS2;X100) 